Results Comparing fast rotating and day shift nurses, significant differences were found in the levels of steroid hormones. Multiple linear regression analysis, considering hormones or vitamin $\mathrm{D}$ as dependent variable and work-shift type as independent variable, showed no differences between the two group as concerns the levels of stress hormones, whereas a significant increase of corticosterone and a marginal decrease of vitamin $\mathrm{D}$ were observed in fast rotating shift nurses, after adjusting for age, body mass index, tobacco smoking, and sampling time.

Conclusion This work shows that the a rapid rotating shiftwork schedule '1-1-1' does not modify the global steroid hormone homeostasis; however, further work is needed to investigate the meaning of the observed increase of corticosterone levels.

\section{MIRNAS IN EXTRACELLULAR VESICLESMEDIATE THE EFFECT OF PARTICULATE MATTER EXPOSURE ON COAGULATION IN A LARGE SAMPLE OF OVERWEIGHT/ OBESE ADULTS}

${ }^{1,2}$ V Bollati* ${ }^{1} \mathrm{M}$ Carugno, ${ }^{1} \mathrm{~L}$ Pergoli, ${ }^{1} \mathrm{~L}$ Cantone ${ }^{1} \mathrm{~S}$ Iodice ${ }^{1} \mathrm{C}$ Favero ${ }^{1} \mathrm{M}$ Hoxha, ${ }^{1} \mathrm{~L}$ Dioni, ${ }^{1} \mathrm{~L}$ Vigna, ${ }^{1,2} \mathrm{M}$ Bonzini, ${ }^{1,2} \mathrm{AC}$ Pesatori. ${ }^{1}$ EPIGET Lab - Dept. Clinical Sciences and Community Health, Università degli Studi di Milano, Milan, Italy; ${ }^{2}$ Fondazione IRCCS Ca' Granda Ospedale Maggiore Policlinico, Occupational Medicine Unit, Milan, Italy

\subsection{6/oemed-2018-ICOHabstracts. 1134}

Introduction In Italy about $45 \%$ of people aged $\geq 18$ years are overweight/obese and might thus be more susceptible to the adverse health effects of air pollution exposure. Particulate matter $\leq 10 \mu \mathrm{m}$ (PM10) represents a common pollutant of living and working environments and has been associated with increased risk of cardiovascular diseases (CVD) and hypercoagulability. Extracellular vesicles (EV) might play an important role in PM-related CVD, as they can travel in body fluids and transfer miRNAs between cells. We investigated whether PM10 exposure is associated with changes in fibrinogen levels, EV release, and EV-miRNA content in a large sample of overweight/obese adults.

Methods EV concentrations were quantified by nanoparticle tracking analysis and flow cytometry. To identify altered levels of EV-miRNAs, we profiled miRNAs of 883 subjects by the QuantStudio 12K Flex Real Time PCR System. The top 40 EV-miRNAs were validated through custom miRNA plates. Statistical analyses included multiple linear regressions, mediation analysis and bioinformatics analysis.

Results In a sample of 1630 overweight/obese subjects from the SPHERE (Susceptibility to Particle Health Effects, miRNAs and Exosomes) study, short-term exposure to PM10 was associated with increased release of EVs, especially those from monocyte/ macrophage components (CD14+) and platelets (CD61+). Nine EV-miRNAs (let-7c-5p; miR-106a-5p; miR-143-3 p; miR-1855 p; miR-218-5 p; miR-331-3 p; miR-642-5 p; miR-652-3 p; miR-99b-5p) were downregulated in response to PM10 exposure and exhibited putative roles in CVD. Five of these nine EV-miRNAs were mediators in the positive association between PM10 exposure and fibrinogen levels.

Conclusions Our study sheds some light on the potential mechanisms underlying the adverse cardiovascular health effects of air pollution exposure. Our results were obtained in a hypersusceptible population and thus strengthen the relevance of health promotion interventions for both the general public and the working population, as they might be particularly feasible in the workplace.

\section{URINARY 8-0X0-7,8-DIHYDRO-2'-DEOXYGUANOSINE IN TUNISIAN ELECTRIC STEEL FOUNDRY WORKERS EXPOSED TO POLYCYCLIC AROMATIC HYDROCARBONS AND METALS}

${ }^{1} \mathrm{~L}$ Campo*, ${ }^{2} \mathrm{M}$ Hanchi, ${ }^{1} \mathrm{E}$ Polledri, ${ }^{1} \mathrm{~L}$ Olgiati, 'S Sucato, ${ }^{2} \mathrm{D}$ Saidane Mosbahi, 'S Fustinoni. ${ }^{1}$ EPIGET Lab., Department of Clinical Sciences and Community Health, Università degli Studi di Milano and Fondazione Cà Granda, IRCCS Ospedale Maggiore Policlinico, Milan, Italy; ${ }^{2}$ Laboratory of analysis, treatment and recovery of environmental pollutants and products Faculty of Pharmacy - Monastir, Tunisia

\subsection{6/oemed-2018-ICOHabstracts. 1135}

Background Electric steel foundry workers are potentially exposed to several toxic chemicals including polycyclic aromatic hydrocarbons (PAHs) and metals. This study was aimed to assess PAHs and metals exposure in foundry workers and its association with the oxidative DNA damage evaluated as urinary 8-oxo-7,8-dihydro-2'deoxyguanosine (8-oxodG).

Methods Ninety-four male workers from an electric steel foundry entered the study. Sixteen unmetabolized PAHs (U-PAHs), 8 hydroxylated $\mathrm{PAH}$ metabolites (OHPAHs), 12 metals and 8oxodG were investigated in urine samples.

Results Among U-PAHs, urinary naphthalene was the most abundant compound, followed by phenanthrene; benzo[a]pyrene level was $<0.30 \mathrm{ng} / \mathrm{L}$. Median 1-hydroxypyrene (1OHPYR) was $0.52 \mu \mathrm{g} / \mathrm{L}$. Job title was a significant determinant for almost all U-PAHs and metals: employees in the steel smelter workshop had higher levels of high-boiling U-PAHs, maintenance workers and workers from the galvanization and rolling workshop were the most exposed to metals. Median 8oxodG level was $3.20 \mu \mathrm{g} / \mathrm{L}$. No correlation between 8 -oxodG and 1-OHPYR or any OHPAH was found. Significant correlations between 8-oxodG and some U-PAHs and metals were found, particularly acenaphthylene, phenanthrene, anthracene, fluoranthene, pyrene, chromium, manganese, cobalt, zinc, arsenic, barium, thallium, and lead.

Conclusions The oxidative DNA damage was moderate and in the range reported in other occupational fields and in the general population. These results indicate that the investigated biomarkers were only minor contributors to urinary 8 -oxodG.

\section{CLOSING THE GAPS BETWEEN OCCUPATIONAL AND ENVIRONMENTAL EXPOSURES AND HUMAN HEALTH}

${ }^{1}$ Balazs Adam*, ${ }^{2}$ Paul TJ Scheepers, ${ }^{3}$ Vivi Schlünssen, ${ }^{4}$ Karel Van Damme, ${ }^{5}$ Claudia Bolognesi, ${ }^{6}$ Torben Sigsgaard, ${ }^{7}$ Thomas Göen, ${ }^{8}$ Richard O'Kennedy, ${ }^{4}$ Ludwine Casteleyn, ${ }^{9}$ Lygia Therese Budnik. 'University of Debrecen, Faculty of Public Health, Department of Preventive Medicine, Debrecen, Hungary; ${ }^{2}$ Radboudumc, Radboud Institute for Health Sciences, Nijmegen, the Netherlands; ${ }^{3}$ National Research Centre for the Working Environment, Copenhagen, Denmark; ${ }^{4}$ University of Leuven, Centre for Human Genetics and Centre for Environment and Health, Leuven, Belgium; ${ }^{5}$ National Cancer Institute, San Martino-IST Environmental Carcinogenesis Unit, IRCCS, Genoa, Italy; ${ }^{6}$ Aarhus University, Dept. of Public Health, Section Environment, Occupation and Health, Danish Ramazzini Centre, Aarhus, Denmark; ${ }^{7}$ Friedrich-Alexander-University Erlangen-Nurnberg, Institute and Outpatient Clinic of Occupational, Social and Environmental Medicine, Erlangen, Germany; ${ }^{8}$ Dublin City University, Biomedical Diagnostics Institute, Dublin, Ireland; ${ }^{9}$ University Medical Centre Hamburg-Eppendorf, Institute for Occupational and Maritime Medicine (ZfAM), Division of Translational Toxicology and Immunology, Hamburg, Germany

10.1136/oemed-2018-ICOHabstracts. 1136 